\title{
Aplicación del método de diseño de mezclas en la formulación de un alimento para perros
}

\author{
Dog food formulation applying the mixing design method
}

\author{
Carlos Elías Peñafiel \\ Universidad Nacional Agraria La Molina \\ celiasp@lamolina.edu.pe
}

\author{
Eliana G. Contreras López ${ }^{2}$ \\ Universidad Nacional Mayor de San Marcos Universidad Nacional Mayor de San Marcos \\ econtrerasl@unmsm.edu.pe
}

Ricardo Angel Yuli Posadas ${ }^{4}$

Universidad Nacional Mayor de San Marcos

\section{ABSTRACT}

\section{RESUMEN}

El objetivo del presente trabajo fue formular un alimento para perros similar a uno presente en el mercado. El alimento del mercado tomado como base declaró en su etiqueta los siguientes ingredientes: harina de pollo (mayor a $27 \%$ ), harina de trigo, harina de soya, harina de maíz, azúcar y grasa de pollo. El análisis de los componentes declarado en la etiqueta del alimento base fue: $27,0 \%$ de proteína, $10,0 \%$ de lípidos, 3,0 \% de fibra y $22,0 \%$ de humedad. Estos componentes se usaron como restricciones en la formulación del alimento nuevo. El programa estadístico utilizado fue el Design-Expert ${ }^{\circledR}$ versión 7.0, Stat-Ease, Inc. Se utilizó un diseño de mezclas látice simplex con siete ingredientes y se analizó cuatro respuestas: proteínas, lípidos, fibra y humedad. La fórmula deducida presentó los siguientes porcentajes de los ingredientes: $27,2 \%$ de harina de pollo, 15,2 \% de harina de trigo, 13,7 \% de harina de soya, 18,7 \% de harina de maíz, 1,9\% azúcar, 5,0\% grasa de pollo y $14,3 \%$ de agua. La formulación deducida alcanzó la siguiente composición: $27,0 \%$ de proteínas, 10,0\% de lípidos, 2,2 \% de fibra y $21,9 \%$ de humedad. La harina de pollo en la fórmula deducida alcanzó un $27,16 \%$ (satisfaciendo el requerimiento de que sea mayor a $27 \%$ y sobrepasó el mínimo de proteínas (18\%) recomendado por la Asociación Americana de Oficiales de Control de Alimentación (AAFCO).

Palabras clave: Alimento para perros, diseño de mezclas, optimización.
The aim of this work was to formulate a dog food similar to a known dog food brand currently offered in the market. The ingredients labeled on the based product are: chicken meal (over $27 \%$ ), wheat flour, soy flour, cornmeal, sugar and chicken fat. A components' analysis performed to this dog food showed: $27,0 \%$ protein, 10,0 \% fat, 3,0 \% fiber and $22,0 \%$ moisture. These constituents were used as constraints in the formulation of the new dog food. Data was analyzed using the Design-Expert 7.0, StatEase, Inc. statistical program. A simplex lattice mixing design with seven ingredients was used and four responses were analyzed; proteins, lipids, fiber and moisture. The deduced formulation presented the following ingredients percentages: 27,2 \% chicken meal, 15,2 \% wheat flour, 13,7 \% soybeans, $18,7 \%$ corn flour, 1,9\% sugar, 5,0 \% chicken fat and 14,3\% water. The developed formula reached the following composition: $27,0 \%$ protein, 10,0 \% fat, 2,2 \% fiber and $21,9 \%$ moisture. Chicken meal deduced in the formula reached $27,2 \%$ (satisfying the requirement to be over $27 \%$ ) and exceeded the minimum protein content (18\%) recommended by the Association of American Feed Control Officials (AAFCO).

Keywords: Dog food, mixture design, optimization.

Historial del artículo:

Recibido: 1 de agosto de 2015. Aprobado: 25 de noviembre de 2015. Disponible en línea: 30 de diciembre de 2015

1 Ing. en Industrias Alimentarias, Mg. en Tecnología de Alimentos, Profesor principal del Dpto. de Tecnología de Alimentos y Productos Agropecuarios de la Facultad de Industrias Alimentarias de la Universidad Nacional Agraria La Molina.

2 lng. en Industrias Alimentarias, Docente de la Facultad de Farmacia y Bioquímica de la Universidad Nacional Mayor de San Marcos.

3 lng. en Industrias Alimentarias, Docente de la Facultad de Farmacia y Bioquímica de la Universidad Nacional Mayor de San Marcos.

4 lng. Químico, Docente de la Facultad de Farmacia y Bioquímica de la Universidad Nacional Mayor de San Marcos. 


\section{INTRODUCCIÓN}

El potencial de crecimiento del mercado de alimentos para perros en nuestro país es alto debido a la creciente clase media con tendencia a tener mascotas (1). Hoy en el mercado encontramos una amplia variedad de marcas, desde las más económicas hasta las más costosas, tanto nacionales como importadas con diferentes tipos de insumos y calidades proteicas. Los animales pueden regular su ingesta energética en función de sus necesidades calóricas diarias, si se les permite acceder a una dieta equilibrada y moderadamente palatable, la mayoría de perros consumirán alimentos suficientes para cubrir sus necesidades energéticas diarias, sin superarlas (2).

La alimentación del perro doméstico (Canis familiaris) se elabora con una gran variedad de insumos, según Hodgkinson et al (3) existe una alta variación en la composición de un alimento pues son formulados utilizando muchas materias primas, existiendo grandes variaciones en la calidad de estos ingredientes, que esto es factible aplicando el Método de Diseño de Mezclas. El alimento del mercado tomado como base declaró en su etiqueta los siguientes ingredientes: harina de pollo (mayor a $27 \%$ ), harina de trigo, harina de soya, harina de maíz, azúcar y grasa de pollo y el análisis de los componentes declarados en la etiqueta del alimento base fue: $27,0 \%$ de proteína, 10,0 \% de lípidos, 3,0\% de fibra y $22,0 \%$ de humedad.

El diseño experimental de mezclas permite aplicar el criterio que la suma de las proporciones de los componentes es el $100 \%$ y la modificación de un porcentaje afecta los otros, en consecuencia, los factores experimentales son los componentes de la mezcla; y los resultados son funciones de tales proporciones y pueden optimizarse mediante la técnica del diseño de mezclas (5). López-Torres et al (6) sostiene que los puntos de un diseño látice simplex están distribuidos uniformemente sobre toda la región simplex. Un látice simplex para q componentes está asociado a un modelo polinomial de grado $m$, éste se denota como un diseño látice simplex $\{q, m\}$. Las

Tabla N ${ }^{\circ}$ 1: Composición de los componentes principales.

\begin{tabular}{lccccccc}
\hline & $\mathbf{1}$ & $\mathbf{2}$ & $\mathbf{3}$ & $\mathbf{4}$ & $\mathbf{5}$ & $\mathbf{6}$ & $\mathbf{7}$ \\
\hline Componentes & $\begin{array}{c}\text { Humedad } \\
\mathbf{g}\end{array}$ & $\begin{array}{c}\text { Prot. } \\
\mathbf{g}\end{array}$ & $\begin{array}{c}\text { Lípidos } \\
\mathbf{g}\end{array}$ & $\begin{array}{c}\text { Carboh. } \\
\mathbf{g}\end{array}$ & $\begin{array}{c}\text { Fibra } \\
\mathbf{g}\end{array}$ & $\begin{array}{c}\text { Cenizas } \\
\mathbf{g}\end{array}$ & $\begin{array}{c}\text { Almidón } \\
\mathbf{g}\end{array}$ \\
\hline Harina de pollo & 9,0 & 63,0 & 15,0 & 3,0 & 2,5 & 7,5 & 0,0 \\
Harina de trigo & 10,0 & 10,5 & 2,0 & 75,6 & 1,5 & 0,4 & 75,6 \\
Harina de soya & 8,0 & 47,0 & 1,8 & 38,9 & 4,3 & 0,0 & 38,9 \\
Harina de maíz & 10,6 & 8,7 & 6,0 & 69,1 & 3,9 & 1,7 & 69,1 \\
Azúcar refinada & 0,6 & 0,0 & 0,0 & 99,2 & 0,0 & 0,2 & 0,0 \\
Tejido graso & 12,0 & 3,0 & 85,0 & 0,0 & 0,0 & 0,0 & 0,0 \\
Agua & 100,0 & 0,0 & 0,0 & 0,0 & 0,0 & 0,0 & 0,0 \\
\hline
\end{tabular}

especialmente en términos de los contenidos de energía y proteína y las formulaciones de muchos alimentos para perros se basan en lograr un bajo costo, por lo cual pueden modificarse los ingredientes o sus porcentajes de participación en las mezclas cuando existen cambios en los precios de estas materias primas. Dentro de los insumos proteicos de origen animal empleados en la alimentación comercial de perros están la carne bovina, ovina y de pollo (4). La harina de pollo está compuesta de restos de aves no consumibles por el hombre, además de plumas, uñas, buche, intestinos y huesos. Las plumas tienen un $87 \%$ de proteínas, pero son de baja digestibilidad, aunque la calidad puede ser mejorada por medios físicos o químicos (4).

Para el desarrollo de nuestra investigación, se tomaron los datos de la composición de un alimento comercial para perros informado en la etiqueta con el objetivo de determinar los porcentajes de los ingredientes de la fórmula que cumplan con aportar los niveles nutricionales requeridos, partiendo de la hipótesis de proporciones para cada uno de los q componentes son los $(m+1)$ valores igualmente espaciados de 0 a 1 definidos como:

$$
X_{i}=0, \frac{1}{m}, \frac{2}{m}, \ldots, 1 ; i=1,2, \ldots, q
$$

Todas las posibles combinaciones de las proporciones que sumen la unidad definen las mezclas o los puntos utilizados en el diseño.

\section{MATERIAL Y MÉTODOS}

Software: Programa Design-Expert 7.0, Stat-Ease, Inc. para el diseño de mezclas.

Diseño experimental: Se utilizó un diseño de mezclas látice simplex, para ello se evaluaron siete ingredientes (harina de pollo, harina de trigo, harina de soya, harina de maíz, azúcar, tejido graso, agua), los mismos 
que representaron las variables independientes (componentes), siendo las variables dependientes (respuesta): proteínas, lípidos, fibra y humedad.

Tabla de composición de alimentos: se elaboró la tabla 1, en la que se puede observar la composición de los componentes principales (7).

\section{RESULTADOS}

Componentes principales y secundarios: Los componentes de la formulación a definir se dividieron en componentes principales y componentes secundarios. En la tabla 1 se observa la composición de cada componente principal del alimento, los mismos que sirvieron para elaborar la tabla 2 que determina el aporte de cada una de las cuarenta y un formulaciones que arrojó el programa con el modelo látice simplex de siete componentes. Los componentes secundarios fueron definidos en función de los límites recomendados y atendiendo lo especificado en la legislación vigente, determinándose un porcentaje de 4,049, hallándose por diferencia el porcentaje de

Tabla $N^{\circ}$ 2: Porcentaje de ingredientes que componen las formulaciones y sus aportes de proteínas, lípidos, fibra y humedad.

\begin{tabular}{|c|c|c|c|c|c|c|c|c|c|c|c|}
\hline \multicolumn{8}{|c|}{ Componentes } & \multicolumn{4}{|c|}{ Respuestas } \\
\hline & 1 & 2 & 3 & 4 & 5 & 6 & 7 & 1 & 2 & 3 & 4 \\
\hline & $\begin{array}{c}\mathrm{H} . \\
\text { pollo }\end{array}$ & $\begin{array}{l}\text { H. de } \\
\text { trigo }\end{array}$ & $\begin{array}{c}\mathrm{H} . \\
\text { soya }\end{array}$ & $\begin{array}{c}\mathrm{H} . \\
\text { maíz }\end{array}$ & Azúcar & $\begin{array}{c}\text { Tej. } \\
\text { graso }\end{array}$ & Agua & $\begin{array}{c}\text { Proteí- } \\
\text { nas }\end{array}$ & Lípidos & Fibra & Humed. \\
\hline 1 & 7,143 & 7,143 & 7,143 & 7,143 & 7,143 & 57,143 & 7,143 & 10,943 & 50,343 & 0,871 & 16,729 \\
\hline 2 & 0,000 & 50,000 & 50,000 & 0,000 & 0,000 & 0,000 & 0,000 & 28,750 & 1,900 & 2,900 & 9,000 \\
\hline 3 & 50,000 & 0,000 & 0,000 & 0,000 & 0,000 & 0,000 & 50,000 & 31,500 & 7,500 & 1,250 & 54,500 \\
\hline 4 & 50,000 & 0,000 & 0,000 & 0,000 & 0,000 & 50,000 & 0,000 & 33,000 & 50,000 & 1,250 & 10,500 \\
\hline 5 & 0,000 & 100,000 & 0,000 & 0,000 & 0.000 & 0,000 & 0,000 & 10,500 & & 1,500 & 10,000 \\
\hline 6 & 0,000 & 0,000 & 0,000 & 0,000 & 0,000 & 50,000 & 50,000 & 1,500 & & & 56,000 \\
\hline 7 & 57,143 & 7,143 & 7,143 & 7,143 & 7,143 & 7,143 & 7,143 & 40,943 & & & 15,229 \\
\hline 8 & 0,000 & 0,000 & 50,000 & 0,000 & 0,000 & 50,000 & 0,000 & 25,000 & 43,400 & 2,150 & 10,000 \\
\hline 9 & 50,000 & 0,000 & 0,000 & 50,000 & 0,000 & 0,000 & 0,000 & 35,850 & 10,500 & 3,200 & 9,800 \\
\hline 10 & 0,000 & 0,000 & 0,000 & 0,000 & 0,000 & 100,000 & 0,000 & 3,000 & 85,000 & 0,000 & 12,000 \\
\hline 11 & 0,000 & 0,000 & 0,000 & 0,000 & 0,000 & 100,000 & 0,000 & 3,000 & 85,000 & 0,000 & 12,000 \\
\hline 12 & 7,143 & 57,143 & 7,143 & 7,143 & 7,143 & 7,143 & 7,143 & 14,693 & 8,843 & 1,621 & 15,729 \\
\hline 13 & 0,000 & 0,000 & 50,000 & 0,000 & 50,000 & 0,000 & 0,000 & 23,500 & 0,900 & 2,150 & 4,300 \\
\hline 14 & 0,000 & 0,000 & 0,000 & 0,000 & 100,000 & 0,000 & 0,000 & 0,000 & 0,000 & 0,000 & 0,600 \\
\hline 15 & 0,000 & 0,000 & 0,000 & 100,000 & 0,000 & 0,000 & 0,000 & 8,700 & 6,0 & 3,900 & 10,600 \\
\hline 16 & 0,000 & 0,000 & 0,000 & 0,000 & 50,000 & 0,000 & 50,000 & 0,000 & & 0,000 & 50,300 \\
\hline 17 & 0,000 & 50,000 & 0,000 & 50,000 & 0,000 & 0,000 & 0,000 & 9,600 & & & 10,300 \\
\hline 18 & 0,000 & 0,000 & 50,000 & 0,000 & 0,000 & 0,000 & 50,000 & 23,500 & & & \\
\hline 19 & 0,000 & 0,000 & 100,000 & 0,000 & 0,000 & 0,000 & 0,000 & 47,000 & & & 000 \\
\hline 20 & 0,000 & 0,000 & 0,000 & 0,000 & 50,000 & 50,000 & 0,0 & 1,500 & & 0,000 & 6,300 \\
\hline 21 & 7,143 & 7,143 & 7,143 & 57,143 & 7,143 & 7,143 & 7,143 & 13,793 & 10,843 & 2,821 & 16,029 \\
\hline 22 & 7,143 & 7,143 & 7,143 & 7,143 & 57,143 & 7,143 & 7,143 & 9,443 & 7,843 & 0,871 & 11,029 \\
\hline 23 & 14,286 & 14,286 & 14,286 & 14,286 & 14,286 & 14,286 & 14,286 & 18,886 & 15,686 & 1,743 & 21,458 \\
\hline 24 & 0,000 & 0,000 & 100,000 & 0,000 & 0,000 & 0,000 & 0,000 & 47,000 & 1,800 & 4,300 & 8,000 \\
\hline 25 & 0,000 & 50,000 & 0,000 & 0,000 & 50,000 & 0,000 & 0,000 & 5,250 & 1,000 & 0,750 & 5,300 \\
\hline 26 & 50,000 & 0,000 & 0,000 & 0,000 & 50,000 & 0,000 & 0,000 & 31,500 & 7,500 & 1,250 & 4,800 \\
\hline 27 & 7,143 & 7,143 & 57,143 & 7,143 & 7,143 & 7,143 & 7,143 & 32,943 & 8,743 & 3,021 & 14,729 \\
\hline 28 & 50,000 & 50,000 & 0,000 & 0,000 & 0,000 & 0,000 & 0,000 & 36,750 & & & 9,500 \\
\hline 29 & 0,000 & 0,000 & 0,000 & 50,000 & 0,000 & 50,000 & 0,000 & 5,850 & & & 11, \\
\hline 30 & 0,000 & 0,000 & 50,000 & & 0,000 & 0,000 & 0,000 & 27,850 & & & 300 \\
\hline 31 & 0,000 & 0,000 & 0,000 & 50,000 & 50,000 & 0,0 & & 4,350 & & & 600 \\
\hline 32 & 100,000 & 0,000 & 0,000 & 0,000 & 0,000 & 0,000 & 0,0 & 63,000 & 15,000 & 2,500 & 9,000 \\
\hline 33 & 0,000 & 0,000 & 0,000 & 100,000 & 0,000 & 0,000 & 0,000 & 8,700 & 6,000 & 3,900 & 10,600 \\
\hline 34 & 0,000 & 50,000 & 0,000 & 0,000 & 0,000 & 50,000 & 0,000 & 6,750 & 43,500 & 0,750 & 11,000 \\
\hline 35 & 50,000 & 0,000 & 50,000 & 0,000 & 0,000 & 0,000 & 0,000 & 55,000 & 8,400 & 3,400 & 8,500 \\
\hline 36 & 100,000 & 0,000 & 0,000 & 0,000 & 0,000 & 0,000 & 0,000 & 63,000 & 15,000 & 2,500 & 9,000 \\
\hline 37 & 7,143 & 7,143 & 7,143 & 7,143 & 7,143 & 7,143 & 57,143 & 9,443 & 7,843 & 0,871 & 60,729 \\
\hline 38 & 0,000 & 0,000 & 0,000 & 0,000 & 0,000 & 0,000 & 100,000 & 0,000 & 0,000 & 0,000 & 100,000 \\
\hline 39 & 0,000 & 0,000 & 0,000 & 0,000 & 100,000 & 0,000 & 0,000 & 0,000 & 0,000 & 0,000 & 0,600 \\
\hline 40 & 0,000 & 50,000 & 0,000 & 0,000 & 0,000 & 0,000 & 50,000 & 5,250 & 1,000 & 0,750 & 55,000 \\
\hline 41 & 0,000 & 0,000 & 0,000 & 50,000 & 0,000 & 0,000 & 50,000 & 4,350 & 3,000 & 1,950 & 55,300 \\
\hline
\end{tabular}


Tabla N ${ }^{\circ}$ 3: Porcentajes de los pseudocomponentes principales.

\begin{tabular}{clr}
\hline & Pseudocomponentes principales & \multicolumn{1}{c}{$\%$} \\
\hline 1 & Harina de pollo & 28,363 \\
2 & Harina de trigo & 15,865 \\
3 & Harina de soya & 14,286 \\
4 & Harina de maíz & 19,565 \\
5 & Azúcar & 2,000 \\
6 & Tejido graso & 5,200 \\
7 & Agua & 14,922 \\
& & 100,000 \\
\hline
\end{tabular}

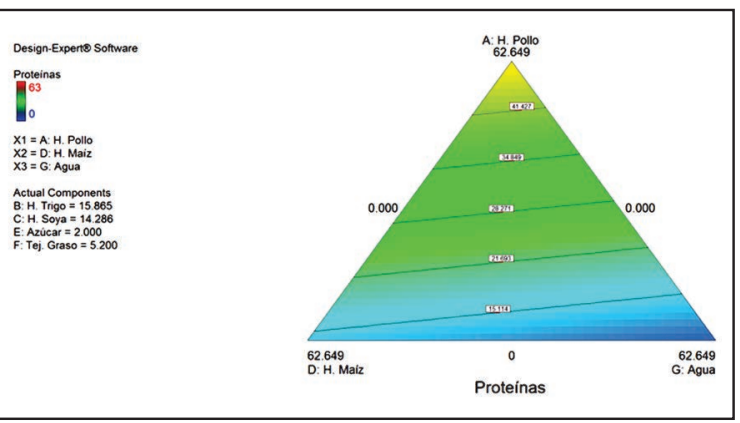

Figura $N^{\circ}$ 1: Gráfico de contornos para las proteínas.

componentes principales $(95,951 \%)$. Se determinó el porcentaje de la harina de pollo bajo la forma de pseudocomponente $(28,66 \%)$ a partir del porcentaje de componentes principales $(95,951 \%)$ y el dato de que la harina de pollo debería tener más de $27 \%$ bajo la forma de componente: $X=27,5 \times(100 / 95,951)$, determinándose así el factor de transformación $\mathrm{f}=$ 100/95,951.

El mismo factor de transformación fue utilizado para hallar el contenido de proteínas, lípidos, fibra y humedad para expresarlos como pseudocomponentes (tabla 3). Estos valores sirvieron de restricciones en el programa de diseño de mezclas.

Componentes ajustados. Los pseudocomponentes

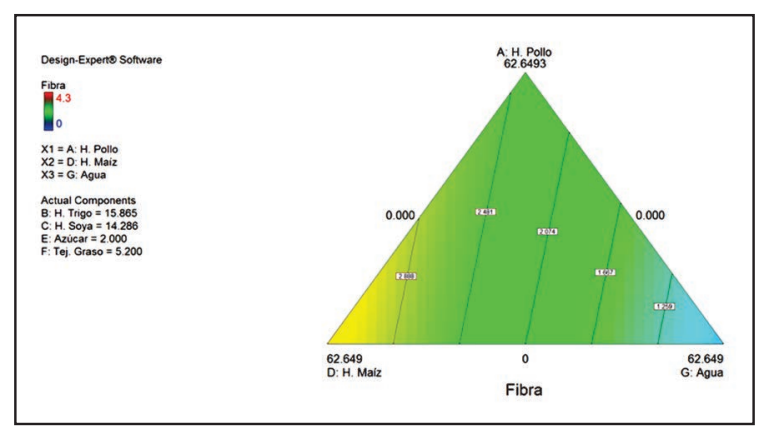

Figura $\mathrm{N}^{\circ}$ 3: Gráfico de contornos para la fibra.
Tabla $N^{\circ}$ 4: Componentes secundarios ajustados.

\begin{tabular}{lcc}
\hline & Componentes & Componentes ajustados \\
\hline Harina de pollo & 27,215 & 27,160 \\
Harina de trigo & 15,223 & 15,192 \\
Harina de soya & 13,708 & 13,680 \\
Harina de maíz & 18,773 & 18,735 \\
Azúcar & 1,919 & 1,915 \\
Tejido graso & 4,989 & 4,979 \\
Agua & 14,318 & 14,289 \\
& 96,144 & 95,951 \\
\hline
\end{tabular}

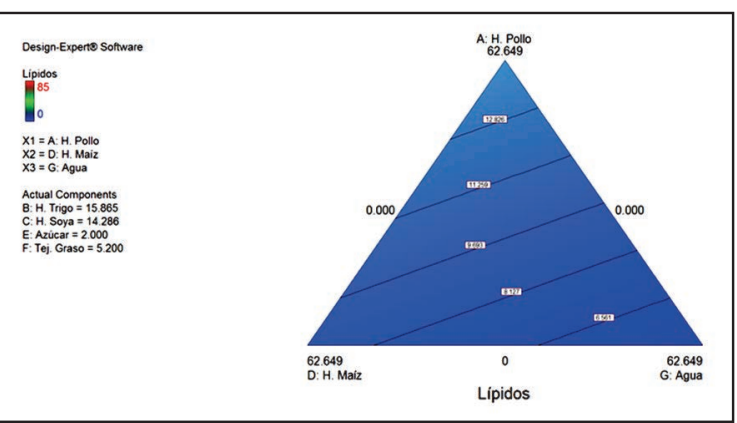

Figura $N^{\circ}$ 2: Gráfico de contornos para los lípidos.

fueron transformados a componentes y ajustados para que, sumen un total de 95,951 (tabla 4) de tal modo que sumados con los componentes secundarios alcancen un total de $100 \%$.

Composición de la fórmula deducida. En la tabla $N^{\circ} 5$ se puede observar los componentes de la formulación deducida y en la tabla $N^{\circ} 6$, la composición que arroja la fórmula deducida, en base a pseudocomponentes y componentes.

\section{Variables de respuesta}

En las figuras 1 al 4 se puede observar los gráficos de contornos de las variables respuesta en las que se

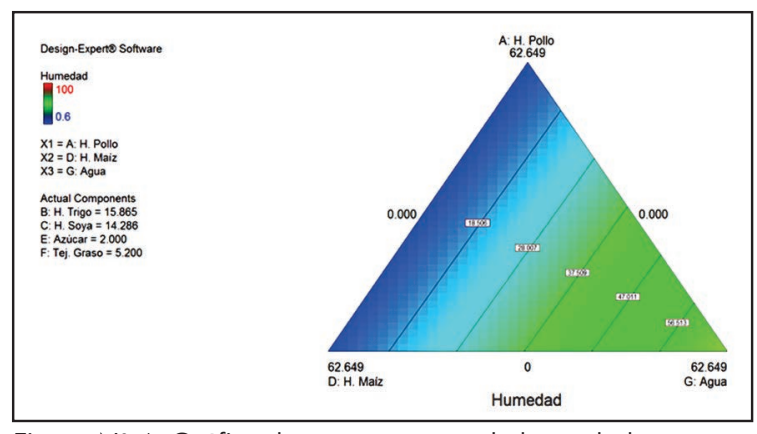

Figura $N^{\circ} 4$ : Gráfico de contornos para la humedad. 
Tabla N ${ }^{\circ}$ 5: Componentes de la formulación deducida.

\begin{tabular}{lrll}
\hline $\begin{array}{c}\text { Componentes } \\
\text { Principales }\end{array}$ & $\%$ & $\begin{array}{l}\text { Componentes } \\
\text { Secundarios }\end{array}$ & $\%$ \\
\hline Harina de pollo & 27,160 & Sal & 1,400 \\
Harina de trigo & 15,192 & Ajo & 0,700 \\
Harina de soya & 13,680 & $\begin{array}{l}\text { Propilenglicol } \\
\text { E-1520 }\end{array}$ & 1,500 \\
Harina de maíz & 18,735 & Humo líquido & 0,040 \\
Azúcar & 1,915 & BHA & 0,009 \\
Tejido graso & 4,979 & Color caramelo & 0,050 \\
Agua & 14,289 & Dióxido de & 0,100 \\
& & titanio E-171 & \\
& & Color amarillo & 0,050 \\
& & Sabor a carne & 0,100 \\
& & Sabor a gallina & 0,100 \\
& 95,951 & & 4,049 \\
\hline
\end{tabular}

Tabla № 6: Composición que arroja la fórmula deducida, en base a pseudocomponentes y componentes.

\begin{tabular}{lccr}
\hline & $\begin{array}{c}\text { En base a los } \\
\text { pseudo } \\
\text { componentes }\end{array}$ & $\begin{array}{c}\text { En base a los } \\
\text { componentes }\end{array}$ & $\begin{array}{c}\text { Recomendado } \\
\text { por AAFCO } \\
\text { (mínimo) }\end{array}$ \\
\hline Proteína & 28,1018 & 27,0 & 18 \\
Lípidos & 10,4172 & 10,0 & 5 \\
Fibra & 2,322 & 2,2 & \\
Humedad & 22,8066 & 21,9 & \\
\hline
\end{tabular}

puede observar los componentes que aportan más proteínas, lípidos, fibra y humedad. En la figura 5 se observa la superficie de respuesta que satisface las restricciones impuestas, así como los porcentajes de los componentes principales de la fórmula deducida con su respectiva composición.

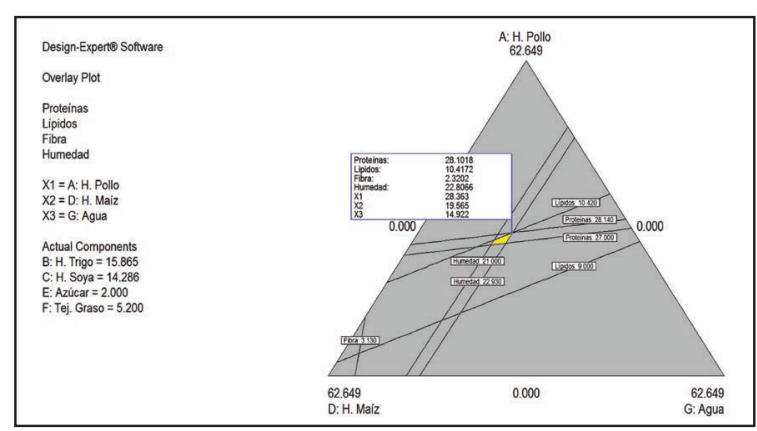

Figura $\mathrm{N}^{\circ}$ 5: Porcentaje de los pseudocomponentes principales.

\section{DISCUSIÓN}

Cada una de las cuarenta y un formulaciones aportó proteínas, en la figura 1 se puede observar su gráfico de contornos como variable de respuesta de la mezcla de harina de pollo, harina de maíz y agua, cuando se mantiene constante los niveles de harina de trigo, harina de soya, azúcar y tejido graso de pollo. El mayor porcentaje de proteína lo aporta la harina de pollo. Silva et al (4) realizaron la evaluación biológica (relación de eficiencia proteica, digestibilidad verdadera, utilización neta de las proteínas y valor biológico) de tres insumos proteicos empleados en la elaboración de alimentos para perros, entre ellos la harina de pollo, resultando esta de mejor calidad proteica que la harina de carne. Respecto a la harina de pollo en la fórmula deducida, se puede observar que ésta es $27,16 \%$, lo que satisface el requerimiento (que sea mayor a $27 \%$ ), que además sobrepasó el mínimo de proteínas (18\%) recomendado por la Asociación Americana de Oficiales de Control de Alimentación (AAFCO) (8).

En la figura 2 se muestra el gráfico de contornos de los lípidos como variable de respuesta de la mezcla de harina de pollo, harina de maíz y agua, cuando se mantiene constante los niveles de harina de trigo, harina de soya, azúcar y tejido graso de pollo. El mayor porcentaje de lípidos lo aporta la harina de pollo, que cumple con los estándares recomendados por la Asociación Americana de Oficiales de Control de Alimentación (AAFCO) para la alimentación de perros adultos (8).

Del gráfico de contornos de la fibra (figura 3), el mayor porcentaje de fibra lo aporta la harina de maíz. Los niveles elevados de fibra pueden disminuir la digestibilidad de otros nutrientes y por tanto reducir la calidad nutricional de la dieta, siendo recomendado del 1,4 al 3,5\% de fibra en alimento para perros (9); los niveles de fibra de la fórmula deducida está dentro del rango recomendado.

En la figura 4 se presenta el gráfico de contornos de la humedad como variable de respuesta de la mezcla de harina de pollo, harina de maíz y agua, cuando se mantiene constante los niveles de harina de trigo, harina de soya, azúcar y tejido graso de pollo.

De acuerdo con el diseño experimental de mezclas, el número total de experimentos fue 41 (tabla 4). Respecto a la composición de la fórmula deducida, los porcentajes de proteínas y lípidos, $27 \%$ y 10 $\%$ respectivamente, fueron exactamente iguales a lo declarado en la etiqueta del alimento base; en relación a la humedad de la fórmula deducida, esta fue un décimo menos en relación la fórmula base y respecto a la fibra de la fórmula deducida ésta fue 2,2 $\%$ frente al 3,0 \% de la fórmula base, diferencia que podría deberse a que los ingredientes de la fórmula base fueron deshidratados con más cáscara que los de la fórmula deducida.

El diseño de mezclas ha sido empleado en el diseño de alimentos $(5,10)$ así como en la formulación de medicamentos (11), siendo eficiente su uso para desarrollar formulaciones nuevas, como es nuestro caso. 
En conclusión, la fórmula deducida presentó los siguientes porcentajes de los ingredientes: $27,2 \%$ de harina de pollo, 15,2 \% de harina de trigo, 13,7\% de harina de soya, 18,7 \% de harina de maíz, 1,9\% azúcar, $5,0 \%$ grasa de pollo y $14,3 \%$ de agua, con la siguiente composición: $27,0 \%$ de proteínas, $10,0 \%$ de lípidos, 2,2\% de fibra y $21,9 \%$ de humedad.

El método de diseño de mezclas es una importante herramienta para deducir formulaciones, tomando como referencia solamente lo declarado en la etiqueta del alimento base.

\section{REFERENCIAS BIBLIOGRÁFICAS}

1. La Prensa.pe. [Online]; 2014 [cited 2015 febrero 03]. Available from: http://laprensa.peru.com/ economia/noticia-peruanos-gastan-us 1228 millones-al-ano-comida-mascotas-23105.

2. Carey DP. Nutrición canina y felina: guía para profesionales de los animales de compañía. 2nd ed. Madrid: Ediciones Harcourt S.A; 2001.

3. Hodgkinson SM, Rosales CE, Alomar D, Boroschek D. Evaluación químico-nutricional de alimentos secos comerciales en Chile para perros adultos en mantención. Archivos de medicina veterinaria. 2004; 36(2): p. 173-181.

4. Silva W, Arbaiza T, Carcelén F, Lucas O. Evaluación biológica en ratas de laboratorio (Rattusnorvegicus) de fuentes proteicas usadas en alimentos comerciales para perros. Revista de
Investigaciones Veterinarias del Perú. 2003; 14(1): p. 18-23.

5. Saltos H, Bayas A. Aplicación de un diseño experimental de mezclas en el desarrollo de una barra energética con base en el Salvado de Palmito de Pejibaye (Bactris gasipaes H.B.K). Revista Tecnológica de la Facultad de Ciencia e Ingeniería en Alimentos. 2010; 23(2): p. 1-8.

6. López-Torres R, Ramírez-Guzmán M, GonzálezCossio F, Martínez-Garza A, Espinosa-Solares T. Una región de confianza para las variables explicatorias en experimentos con mezclas utilizando calibración. Agrociencia. 2002; 36(5): p. 579-592.

7. Reyes M, Gómez-Sánchez I, Espinoza C, Bravo F, Ganoza L. Tablas peruanas de composición de alimentos. 8th ed. Lima: Instituto Nacional de Salud; 2009.

8. Peteducation.com. [Online].; 2015 [cited 2015 febrero 03]. Available from: http: //www.peteducation.com/article. $\mathrm{cfm}^{2} \mathrm{c}=2+1659$ \&aid $=662$.

9. Eukanuba. Fibra un componente importante de la dieta para perros y gatos (II). Animalia. 2004; 17(166): p. 67

10. Millán F, Ramírez J. Diseño de un texturizado multisápido a base de frutas tropicales y biopolímeros de algas. Anales de la Universidad Metropolitana de Venezuela. 2012; 12(2): p. 189206.

11. Pinillos J, Lopera C. Elaboración de una formulación farmacéutica a través de un diseño experimental de mezclas. Vitae. 2009; 16(3): p. 338-353. 